\title{
IMPROVING THE TRANSFER OF MEDICALLY COMPLEX PATIENTS FROM THE CARDIAC INTENSIVE CARE UNIT TO THE CARDIOLOGY INPATIENT WARD
}

Jean Storey, Laurie Mustin, Nicolas Madsen, Melissa Kimball, Gina Schoenling, James Brown, Jonathan Byrnes, David Cooper. Cincinnati Children's Hospital Medical Center, United States

\subsection{6/bmjqs-2015-IHlabstracts.25}

Background Readmission to the cardiac intensive care unit (CICU) increases LOS and cost, negatively impacts patient experience, throughput and resource allocation. A review of unplanned readmissions to the CICU identified that transfer of care and monitoring practices were not differentiated based on patient specific risk.

Objectives To increase days since last readmission to the CICU within 48 hours of transfer by defining patients at increased risk and standardizing key transfer processes.

KEY DRIVER DIAGRAM

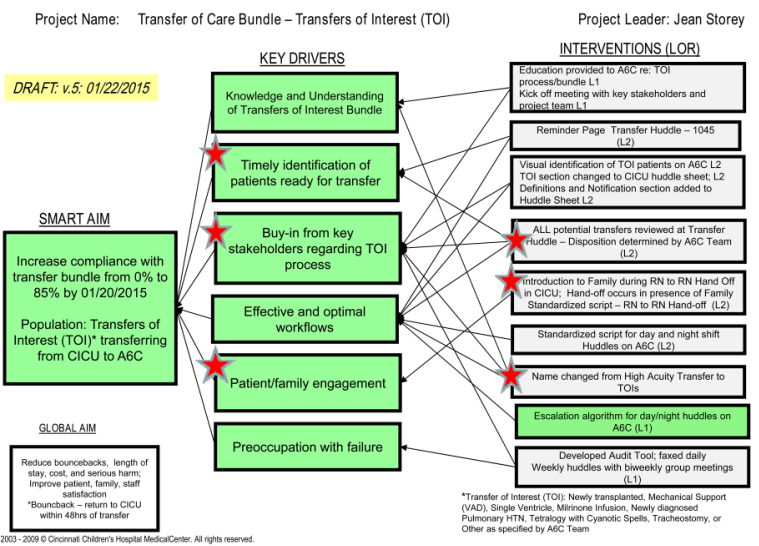




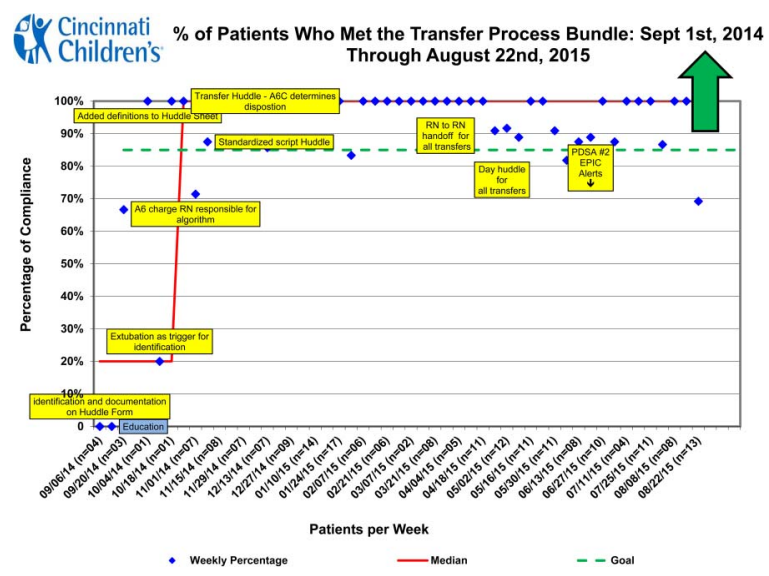

Figure 2

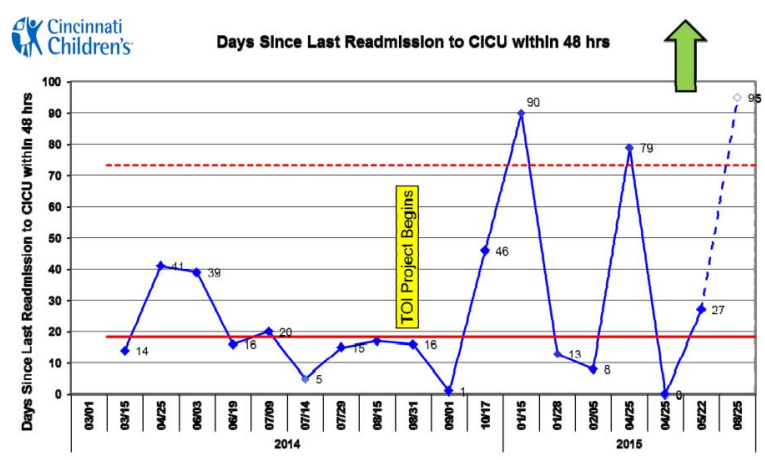

Figure 3

Methods A standardized care process was developed and tested for patients at increased risk of readmission to CICU within 48 hours of transfer. Bundle components included: Patients identified 24 hours prior to transfer; Face to face nurse handoff in the CICU; Standardized team huddle on arrival to ward and prior to overnight hand-off; VS every 2 hours, I/O every 4 hours for 24 hours and RN staffing ratio of 2:1. Statistical process control charts were used to monitor progress.

Results Transfer bundle compliance reached a median of $100 \%$. Days between 48 hour readmissions to CICU increased from an average of 12 days to 45 days. Process control charts demonstrate 3 data points considered special cause after bundle compliance reached $100 \%$.

Conclusions Transfer of care processes and outcomes for patients at increased risk of CICU readmission have improved. These interventions can be spread to other hospital units with the hopes of achieving similar results. 